\title{
Predictive modeling of chlorophyll a for tropical lake by means of hybrid evolutionary algorithm (HEA)
}

\begin{abstract}
This paper discusses the application of Hybrid Evolutionary Algorithm (HEA) model to simulate dynamics of Chlorophyll a in Lake Putrajaya, Malaysia for 7-days-ahead prediction using data from 2003 until 2008. Results are measured in term of closeness between predicted and measured data as well as the root mean square error value (RMSE) and rsquare. The HEA achieved reasonable accuracy in predicting timing and magnitudes of algal blooms. Chlorophyll a concentrations levels at Lake Putrajaya were divided into low and high abundance in the water column depending on the $\mathrm{pH}$ threshold value of 7.9. Chl-a concentrations in Lake Putrajaya is predicted higher if $\mathrm{pH}$ of the water exceeds 7.9. Sensitivity analyses revealed that an optimal condition for algal growth and abundance is not only driven by physical and chemicals characteristics of the water body but also by impact of the monsoon season where a highest Secchi depth of up to $2.4 \mathrm{~m}$ was observed. The HEA has shown potential for utilisation in early warning and strategic control of algal blooms in tropical freshwater lake. Outcomes of this research offers an original contribution to the knowledge domain Ecology of Tropical Lakes by successfully applying data driven models (HEA).
\end{abstract}

Keyword: Tropical lakes; Hybrid evolutionary algorithm (HEA); Chlorophyll-a; Eutrophication 